Volume 8

July 1981

\title{
A Peer Counseling Training Program For Disabled Women: A Tool for Social and Individual Change
}

Marsha Saxton

Boston Self Help Center

Follow this and additional works at: https://scholarworks.wmich.edu/jssw

Part of the Clinical and Medical Social Work Commons, and the Social Work Commons

\section{Recommended Citation}

Saxton, Marsha (1981) "A Peer Counseling Training Program For Disabled Women: A Tool for Social and Individual Change," The Journal of Sociology \& Social Welfare: Vol. 8 : Iss. 2 , Article 9.

Available at: https://scholarworks.wmich.edu/jssw/vol8/iss2/9 


\title{
A PEER COUNSELING TRAINING PROGRAM FOR DISABLED WOMEN
}

\author{
A Tool for Social and Individual Change \\ Marsha Saxton \\ Boston Self Help Center \\ Boston, Mass.
}

\section{ABSTRACT}

This article presents a description of a successful peer counseling training program for disabled women, offered in a community-based seif help organization. The program provides both training in peer counseling skills, and a personal growth experience. It was carried out in a group context specifically designed for the needs of women with disabilities. The counseling approach, the training format and curriculum, and the evaluation procedures can all serve as a basis for agencies interested in developing similar programs.

\section{Introduction}

The women's movement of the 60's and 70's taught us of the potential of group activities by and for women. Women's groups have provided a strong counter-force to oppression, and have led to the empowerment of the individual. The success of the book, Our Bodies, Ourselves 1 , gives testimony to the power of self help for women.

Disabled women, like their able-bodied sisters can benefit greatly from peer groups. Being female and disabled in our culture often means experiencing the overlap of two kinds of oppression. Together they of ten lead to a double measure of isolation and powerlessness. A disabled woman in a peer group can realize she is not alone in her feelings about her unique body and uncommon life circumstances. She can meet and learn from others who have confronted similar experiences, and she can look forward to change. A peer counseling training group can be a particularly powerful forum for this process. In such a group, by building on their own experiences participants acquire and share valuable counseling skills for use in assisting each other's growth. 
Thus, the purpose of the group is dual: to train participants in the use of counseling skills, and to provide a therapeutic group experience. This paper presents a model of a peer counseling training program for women with disabilities, offered in a community-based self help organization called the Boston Self Help Center.

\section{Disabled Women}

The concerns of disabled women are unique and deserve special consideration. The cultural interactions between sexism and the oppression of disabled persons (or "able-bodiedism") serve to reinforce each other in the personality of the individual. For example, sexism in this culture perpetuates traits of passivity, dependence and childishness in women. These traits disuade women from excelling. Able-bodiedism produces the same effects and similarly results in a reduced likelihood of success. The cultural standards of physical attractiveness exacerbate this situation. Women in this culture are beset with messages from the media to buy products to alter aspects of their appearance which do not conform to established standards of beauty. Disabled people similarly are made to feel that they must hide their bodies. Disabled women who do not conform to cultural standards of beauty are doubly stigmatized.

Thus the issue of sexuality can often present difficulties for disabled women. The problems stem from two sources: those problems which are a result of actual physical limitations, and those which are a result of myths and attitudes. Many of the cultural assumptions regarding sexuality are particularly hurtful to disabled people. These include such societal "rules" as:

--sex shouldn't be discussed or planned, but should be "spontaneous", --sexual intercourse and orgasm are necessary for sexual satisfaction.

These assumptions may lead to a sense of failure for women with physical limitations requiring adaptations, special planning or assistance. These social myths interfere with the realization that sexuality is not limited to specified "acts" or set behaviors. When these beliefs are recognized as myths by the individuals, the problems arising from physical limitations seem much less formidable.

Myths regarding disabled persons include the common assumption that disabled persons are asexual, either because they are incapable of sexual function, or because they just "shouldn't want it." Such social abberrations as homosexuality are of ten considered by society to be particularly bizarre among disabled persons. 
Because of sexism, women's skills and intelligence are down played. In the area of sexuality, women may perceive a chance to redeem themselves as highly valued. Disabled women, however, because of the myths and assumptions regarding sexuality and disability, are not offered this chance at redemption, and therefore may be made to feel particularly worthless. It was interesting to note that some disabled women, self identified as "feminists", have felt a conflict between the goals of feminism, and their personal needs. The feminist movement encourages women to resist being regarded as sexual objects and targets of objectifying sexual comments from men, while many disabled women have never been such targets and whish to "have a turn" at being viewed as a sexval or "sexy" person.

The disabled individual faces many psychological adjustments. Unfortunately, this is of ten made considerably more difficult by the social and attitudinal barriers which the disabled person is likely to encounter among family, friends and others.

The disabled person may face the gamut of behaviors from the ablebodied world ranging from gawking to avoidance, from pity to resentment, or from vastly lowered expectations to awe. Along with these attitudes, disabled persons confront a variety of tangible barriers: architectural inaccessability, lack of interpreters services for deaf persons, and lack of Brailled or taped materials for blind persons. In addition, disabled persons, particularly females, confront less tangible barriers: discrimination in employment, second class education, and restricted opportunities for full participation in the political life of the community. Consequently, the disabled woman may of ten experience feelings of self-hate, hopelessness, isolation, unworthiness or ugliness.

\section{Peer Counseling}

A peer counselor is in a unique position to assist another disabled person in dealing with life issues. Peer counseling is a process by which one person is helped by another person who has had similar or related experiences. The concept and practice of peer counseling is gaining increased attention. Professionals in a variety of helping fields are beginning to recognize and utilize this effective resource. Egan, in The Skilled Helper? notes:

"There is a growing body of opinions and evidence...that helpers with extensive training in psychological theory and a variety of academic credentials do not necessarily help, and that the para-professional helper, if properly 
trained in helping skills, can become very effective even without extensive training in psychological theory."

Peer counseling has gained special significance in the field of rehabilitation. Title VII of Public Law 95-602 (The 1978 Rehabilitation Amendments) provides statutory authority for the use of peer counselors in Independent Living Centers. The purpose of these centers is to train severly disabled persons in attaining the maximum degree of independence needed to live in a community-based setting. The new mandate is in large part a result of a growing disability rights movement demanding greater consumer involvement in the provision of human services.

The practice of peer counseling can address many of the issues facing disabled persons involved in the rehabilitation process. A useful definition offered by Schatzlein: 3

Peer counseling is a necessary adjunct to the rehabilitation process, in which a severely disabled person who has made a successful transition from institutional to independent community living, provides resource information, support, understanding and direction to another disabled person who desires to make a similar transition.

Frequently, the peer counselor will offer training in adapting to the physical demands of a disability. For example, the peer counselor may assist a peer client with wheelchair mobility or wheelchair maintenance. The peer counselor may also share knowledge and experience in coping with the broader aspects of disability, such as obtaining social security benefits from governmental agencies or locating accessible housing and transportation.

The peer counselor can also be instrumental in helping an individual confront various emotional aspects of disability. The approach described here emphasized the psycho-social aspects of disability. The assumption here is that an individuals' ability to function in the psycho-social sphere often shapes his or her ability to cope with the various aspects of dealing with a disability. This may include one's ability to perform medical and non-medical self-care, revise vociational goals, and negotiate reactions of family and friends to one's disability.

\section{A Model Program}

The following is a description of the peer counseling training 
program for disabled women held at the Boston Self Help Center (BSHC). The purpose of the group was to provide training in peer counseling skills as well as a personal growth experience.

The theoretical basis for this peer counseling program was.derived primarily from the works of Robert Carkhuff. 4 Simply stated, the counselor facilitates an atmosphere of trust and genuine respect where the client can express feelings without fear of judgement on the part of the counselor. It is assumed that clear thinking and decisive action will result after the client has had the opportunity to openly ventilate fears, anger, grief, and other emotions. 1 t is also assumed that maximum growth occurs when responsibility for life decisions are made by the client him/herself. Thus, the counselor rarely offers advice or gives interpretation.

This particular counseling approach views the individual as the target of numerous emotional social hurts. These hurts begin at an early age and serve to create self-limiting behavior patterns. The inidvidual can recover from these distresses, and regain a more rational grasp of his or her life, if given the necessary emotional support and resource.

One significant aspect of this approach is the concept of oppression, here defined as "the systematic invalidation of one social group by another." Participants are encouraged to examine the effects of oppression, (not only sexism and able-bodiedism, but other types such as racism and classism) on their own personalities. In group discussions, participants identify cultural myths and stereotypes, focusing on those aimed at them personally. Participants realize that the cultural messages are often so strong that individuals accept them as truth and operate to perpetuate the myths. The reversal of this "internalized oppression" is a major goal of the training.

The nature and limitations of the peer counseling relationship are clearly defined in the training. Basically, the peer counselor is expected to meet regularly with the client to provide a setting where the client may discuss ideas, express feelings, set goals and report back on them. The peer counselor is not expected to solve the clients problems, socialize with the client, or attempt to meet the client's personal needs.

Peer counselors are trained to determine the difference between life issues which are manageable in the peer counseling context, and those which are not. If they are not, the client is referred to an appropriate professional. 
The peer counseling training group was run by a leader and an assistant leader, both disabled women, who are staff counselors at the Boston Self Help Center. The group leader (this author) developed the training program and the assistant leader was trained as a peer counselor and group leader in this program.

The training group was planned for an initial twelve week period, with continuation for additional time negotiable with leaders and participants. At the time of this writing the group is in its eighteenth week.

The group was advertised in the BSHC's schedule-of-events mailing. Prospective participants were interviewed by one of the group leaders. The interview assessed the applicant's ability to:

--acquire and utilize the counseling theory and techniques;

--form relationships;

--communicate caring with other group members;

- follow through in attending group sessions and practicums; and

--actively utilize peer counseling skills in some capacity following the completion of the group.

Four of the participants group fees were funded by a grant from the Massachusetts Rehabilitation Commission. The others were charged on a sliding scale basis depending on income.

The training group was cross-disability in composition, which afforded participants exposure to a wide variety of disability types, yet with focus of the commonalities of life experiences. This is consistent with BSHC's philosophy that alliance with differing interest groups will result in effective social change for all.

The group was composed of eight women, including two leaders and ranged in age from 25 to 42 years. All were disabled, (including the following disabilities: cerebral palsy, arthritis, post polio, spina bifida, visual impairment, upper-extremity amputation) were white, and of lower middle to middle class. Five participants were employed, either part or full time, one in college and two unemployed. Four members had had some type of emotional counseling or therapy prior to this group.

The group leaders, by functioning as participants, helped create an atmosphere of trust and peerness, enabled the leaders to model the role of "active client", and encouraged openness and risk-taking in sharing of feelings. 
Evaluation questionnaires were administered to participants following twelve weeks of group meetings, asking for evaluation of the group along the following parameters:

--effectiveness of teaching skills of leaders;

- impact of the group on personal growth, independent living skills and vocational raadiness; and,

--effectiveness of the counseling approach, particularly as related to being female and being disabled.

\section{Training Curriculum}

The training format of the two hour weekly training sessions typically included:

--lecture and discussion on weekly topics;

--demonstrations of counseling techniques before the group;

--short practice sessions;

--feedback and discussion about the practicum counseling sessions;

--personal sharing by group participants.

The practicum counseling sessions held between pairs of group members were scheduled outside of group time. These served to reinforce skills learned, and allowed each individual to take a turn as "counselor" and as "client". A portion of every training group session was devoted to supervision of the practicum counseling sessions.

The following is a description of the curriculum for the first twelve weeks of the group:

Session One was devoted to introduction of members and explanations of basic theory used in the peer counseling program. Participants were given the opportunity to explore and identify areas of their own lives they wanted to focus on in the practicum sessions.

Sessions Two and Three focused on counseling techniques, including active listening, role-playing and the use of fantasy.

Session four explored the use of self-appreciation validation of others and means of exposing and contradicting feelings of inadequacy. The need for warm, open expression of respect, affection and appreciation of the peer $c$ lient in individual sessions as well as in the training group, was stressed. 
Session Five emphasized aspects of body image and loss as they relate to disability and chronic disease. Here, discussion focused on societal standards of acceptable appearance and the influence of the "Youth and Beauty" cultural orientation on self-image of disabled women. Counseling techniques eliciting self-appreciation and body awareness were presented.

Session six explored the nature of cultural oppression of disabled persons, including aspects of discrimination, stereotypes, etc. Emphasis was placed on identifying ways disabled persons internalize these cultural messages and the use of counseling techniques to contradict this.

Sessions Seven and Eight focused on the impact of sexism on women and in particular disabled women. Counseling techniques were demonstrated to expose and contradict internalized feelings of passivity, powerlessness, unworthiness, etc. The interaction of the effects of sexism and the oppression of disabled persons was discussed.

Session Nine focused on Independent Living. Goal setting and problem solving techniques were presented as ways to overcome barriers to maximal self-reliance for disabled women. Community resources were reviewed here.

Sessions Ten and Eleven focused on sexuality and sexual relationships. Here sexuality was linked to issues previously discussed, including sexism and stereotypes regarding disability. Participants were encouraged to share how early childhood training as well as experiences in relationships influenced sexual selfimage.

Session Twelve focused on overcoming feelings of isolation in male and female relationships. Participants set goals to overcome barriers in achieving full personal and social lives.

\section{Participants Reactions}

The peer counseling skills gained in this program provided each individual with a sense of effectively assisting others in areas where she herself had experienced difficulties. She could now draw upon her experiences as a means of offering support. This sense of being able to assist others is extremely important for severely disabled persons, because it opposes the cultural notion that people with disabilities 
have little to offer. Several individuals noted that the tone of the group, that of "moving toward social change", helped participants feel that they were learning valuable skills for use in the disability rights movement, and not just "making people feel better". Stated one group nember:

"I feel that my abilities as a counselor have grown with each session. The opportunity to observe and participate in counseling sessions in the group, getting feedback from other members and leaders, has been valuable. The weekly 'co-counseling' sessions with one person are invaluable. Being a member of this group has given me more confidence in myself and in my ability to interact on different levels and thus participate more fully in the disabled rights movement. I have worthwhile ideas and thoughts to contribute."

With the increasing use of peer counseling in rehabilitation nationwide, the training allows participants to develop a marketable skill. Two of the women reported that they have taken on new counseling roles in their work places. Another is now working at $85 \mathrm{HC}$ as a peer counselor doing intake interviews and two others are planning to join the BSHC staff as peer counselors in the near future. Group participants who become staff peer counselors are thus realizing the goal of seif help. Many individuals who have undergone the training now work in other agencies and are training other peer counselors. Individuals often use the peer counseling training to explore an interest in counseling as a profession, before undertaking schooling in that field.

A unique and important aspect of the training program at BSHC is that all peer counselors who work at BSHC are themselves members of a support group. This is an integral part of the on-going training and supervision. It is consistent with the BSHC philosophy that effective counselors require continuing support for themselves and their feelings related to work as a counselor.

With respect to personal gains, one of the most prevalent reactions to the group was the realizations that "I'm not alone" in experiencing the feelings that they had encountered. Both the group sessions and the individual peer counseling sessions provided a contradiction to the feelings of isolation that often accompany being disabled. For example, one participant noted:

"I have been made more aware that there are others who face many of the same issues and frustrations that I grapple with. 
This has lessened my feelings of separateness from the mainst ream."

The concept of cultural oppression of both women and disabled persons proved to be a valuable tool for personal growth. Participants reported experiencing a challenge to the feelings of powerlessness and a reversal of their guilt for their "failure to be normal". One participant reported:

"I see more clearly now how some of the problems I have socially, developmentally, etc. are not unique to me and are actually rooted in the systematic oppression that accompanies a disability. The phrase, 'it's not your fault', as it relates to oppression is helping to allay very strong guilt patterns."

Participants revealed a greater acceptance of themselves as disabled women. One stated simply, "l feel much more proud and self accepting, much more." Another said:

"I've begun to see that it's [my disability] not the end of the world. I'm beginning to really believe that 1 'm competent and in control of my life again, in spite of this disability."

Other members shared:

"The group let me explore and examine life's hurts past and present. With the group's help, I've begun the healing process and have begun to grow strong with self-respect and self-assurance."

"My self-image and feelings of inadequacy about being a woman have been changed to the point where I have a more positive feeling about myself as a woman and as a person. Being able to share some long pent-up feelings and getting support helped."

\section{In Conclusion}

This model is by no means restricted in application to disabled women. The reciprocal client-counselor paradigm, as well as the "challenging oppression" focus, can successfully be applied to many persons of various backgrounds.

Significant components of the program which contributed to its success included: 
1. Effective screening of applicants to help ensure maximum benefit from the program;

2. A workable counseling approach, easily acquired and utilized by beginners, with room for expansion of skills by experienced peer counselors;

3. A training curriculum relevant to the needs of the participants, which embodied both personal growth and social change;

4. Strong peer leadership by persons willing to model both the client and counselor roles; and,

5. An immediate channel for peer counselors' energies, which emphasized on-going supervision and support.

In summary, peer counseling training seemed beneficial for disabled women in the following ways:

The approach described here was able to provide a valuable and marketable skill, effectively build the confidence and self-image of participants, contradict patterns of isolation and poweriessness, and allow individuals to successfully set and attain personal goals. Peer counseling can assist individuals in attaining their optimal levels of independence, meeting vocational goals, and developing a positive selfconcept. By helping individuals recognize their full human and legal rights and by challenging culturally oppressive behaviors and attitudes which prevent the realization of their full human potential, this program thus becomes an effective vehicle for furthering the goals of the disability rights movement.

\section{REFERENCES}

Arkridge, R., Means, B., Milligan, T., Farley, R.

1978 Interpersonal Skills Training: Basic Helping Skills for Rehabilitation Workers. Arkansas Rehab. Research and Training Ctr. University of Arkansas, Arkansas Rehab. Services.

Boston Women's Health Book Collective'

1972 Our Bodies, Ourselves. Simon and Schuster, New York. Carkhuff, Robert R.

1969 "Helping and Human Relations." Vol.11, Holt-RinehartWinston, Inc. 270:277.

Carkhuff, R.R., Berensen, B.G. 4

1977 Beyond Counseling and Therapy. Holt-Rinehart-Winston, Inc. 
Corn, R.

1977 Aiding Adjustment to Physical Limitation: A Handbook for Egan 2 Peer Counselors. Howard Community College, Columbia, Md.

1975 The Skilled Helper: A Model for Systematic Helping and Interpersonal Relating. Monterrey, Ca.: Brooks/Cole p.9.

Griffin, E.L., Martin, W.

1979 Peer Counseling: Process and Goal. 1979 National Spinal

Cord Injury Foundation Convention Journal, 31 st Annual Convention, August 5-9, 1979, Denver, Colorado.

Jackins, $H$.

1970 Fundamentals of Co-Counseling Manual. Rational |s land Publishers, Seattle, Wash.

Schatzlein, J.E. 3

1978 Spinal Cord Injury and Peer Counseling/Peer Education. (unpub. paper) Regional Spinal Cord Injury Center, Dept. of Physical Medicine and Rehabilitation, University of Minnesota Hospitals.

Tracy, G.S., Gusson, Z.

1976 "Self-Help Groups, A Grassroots Response to a Need for Services." Journal of Applied Behavioral Science. $12: 381-396$. 